\title{
Analysis of interspecies adherence of oral bacteria using a membrane binding assay coupled with polymerase chain reaction-denaturing gradient gel electrophoresis profiling
}

\author{
Ren-ke Wang ${ }^{1,2}$, Xue-song He ${ }^{1}$, Wei Hu ${ }^{1}$, Renate Lux ${ }^{1}$, Ji-yao Li $^{2}$, Xue-dong Zhou ${ }^{2}$, Wen-yuan Shi ${ }^{1,2} *$ \\ ${ }^{1}$ School of Dentistry, University of California at Los Angeles, CA 90095, USA; ${ }^{2}$ State Key Laboratory of Oral \\ Diseases, West China School of Stomatology, Sichuan University, Chengdu 610041, China
}

Information on co-adherence of different oral bacterial species is important for understanding interspecies interactions within oral microbial community. Current knowledge on this topic is heavily based on pariwise coaggregation of known, cultivable species. In this study, we employed a membrane binding assay coupled with polymerase chain reaction-denaturing gradient gel electrophoresis (PCR-DGGE) to systematically analyze the co-adherence profiles of oral bacterial species, and achieved a more profound knowledge beyond pairwise coaggregation. Two oral bacterial species were selected to serve as "bait": Fusobacterium nucleatum (F. nucleatum) whose ability to adhere to a multitude of oral bacterial species has been extensively studied for pairwise interactions and Streptococcus mutans (S. mutans) whose interacting partners are largely unknown. To enable screening of interacting partner species within bacterial mixtures, cells of the "bait" oral bacterium were immobilized on nitrocellulose membranes which were washed and blocked to p revent unspecific binding. The "prey" bacterial mixtures (including known species or natural saliva samples) were added, unbound cells were washed off after the incubation period and the remaining cells were eluted using $0.2 \mathrm{~mol} \cdot \mathrm{L}^{-1} \mathrm{glycine}$. Genomic DNA was extracted, subjected to 16S rRNA PCR amplification and separation of the resulting PCR products by DGGE. Selected bands were recovered from the gel, sequenced and identified via Nucleotide BLAST searches against different databases. While few bacterial species bound to $S$. mutans, consistent with previous findings $F$. nucleatum adhered to a variety of bacterial species including uncultivable and uncharacterized ones. This new approach can more effectively analyze the co-adherence profiles of oral bacteria, and could facilitate the systematic study of interbacterial binding of oral microbial species.

Keywords: membrane binding assay; polymerase chain reaction-denaturing gradient gel electrophoresis; coaggregation; Fusobacterium nucleatum; Streptococcus mutans

International Journal of Oral Science (2011) 3: 90-97. doi: 10.4248/IJOS11033

\section{Introduction}

More than 600 bacterial species inhabit the human oral

*Correspondence: Wen-yuan Shi

Tel: 001310825 8356; Fax: 0013107947109

E-mail:wenyuan@ucla.edu

Received 8 March 2011; Accepted 18 March 2011 cavity [1-3] in highly organized communities commonly known as dental plaque. These dental plaque communities [4] are responsible for many oral infectious diseases including dental caries and periodontal disease [5-8] Extensive studies have revealed many of the complex interactions between the residents of the oral microbial community [9-10]. During dental plaque formation, the "early colonizing" bacteria express components that 
enable effective adherence to the target tissues including teeth surface and mucosa [11-12]. Late colonizers adhere to the early colonizers or "bridging organisms" such as Fusobacterium nucleatum (F. nucleatum) to integrate into the community [13]. The ability to adhere to other bacteria species is a fairly common characteristic among oral microbes. It has been implicated to play an important role in the formation of structured multispecies oral biofilms that affect the microbial ecology and pathology of oral cavity [14-15].

Most investigations of oral bacterial interspecies binding have relied on a coaggregation assay developed by Cisar et al. more than 30 years ago in which pairwise combinations of bacteria are tested in planktonic culture [14, 16-18]. A more recent membrane-based binding assay developed by Lamont et al. increased the sensitivity of detecting interbacterial binding by immobilizing one bacterial species on a membrane and using radio-labeled test organism for easy detection and quantification [9]. In this study, we expanded from this previously developed membrane binding assay which was limited to examining one-on-one interactions and used complex salivary samples cultured in the recently reported SHI medium that supports growth of diverse oral communities [19] for screening against the membrane bound "bait" organism. Subsequent polymerase chain reaction-denaturing gradient gel electrophoresis (PCRDGGE) profiling and species identification allowed investigation of the comprehensive co-adherence profiles of F. nucleatum and Streptococcus mutans ( $S$. mutans). We demonstrated that compared with existing assays for studying bacterial co-adherence, this new approach is effective in simultaneous identification of multiple coadherence partners. Furthermore, the increased species diversity achieved by using SHI medium for cultivating oral microbial flora from saliva can be used to discover new co-adherence partners including previously unculturable bacteria.

\section{Materials and Methods}

\section{Bacteria and culture conditions}

Bacterial strains used in this study include S. mutans UA140, Porphyromonas gingivalis (P. gingivalis) W83, F. nucleatum ATCC23726, Streptococcus mitis (S. mitis) ATCC9811, Escherichia coli (E. coli) DH5a. S. mutans, $S$. mitis and E. coli were grown in Brain Heart Infusion (BHI, Difco) medium. F. nucleatum and $P$. gingivalis were grown in Columbia Broth (CB, Difco). E. coli was grown under aerobic condition at $37^{\circ} \mathrm{C}$, while all other bacterial strains were grown in an aerobic atmosphere of $80 \% \mathrm{~N}_{2}, 10 \% \mathrm{CO}_{2}$, and $10 \% \mathrm{H}_{2}$ at $37{ }^{\circ} \mathrm{C}$.

\section{Saliva collection}

Saliva samples were collected from six healthy subjects, age 25-35 years under UCLA-IRB \#09-08-068-02A. Subjects with systemic diseases or taking prescription or non-prescription medication were excluded from the study. Subjects were asked to refrain from any food or drink 2 hours before donating saliva and to spit directly into the saliva collection tube, $5 \mathrm{~mL}$ saliva was collected from each person. Saliva samples were pooled and centrifuged at $2600 \times \mathrm{g}$ for $10 \mathrm{~min}$ to spin down large debris and eukaryotic cells. The supernatant was referred to as pooled saliva and used throughout this study. A $5 \mathrm{~mL}$ subsample of pooled saliva was centrifuged again at $14000 \times \mathrm{g}$ for $5 \mathrm{~min}$ and the pellet was collected for DNA extraction and PCR-DGGE analysis to obtain the bacterial profile of the original saliva. The rest of the pooled saliva was used for planktonic culture in SHI medium [19].

\section{Mice gut microbial samples}

The mice gut microbial samples were recovered from a lab stock that was described in a previous study [20].

\section{Membrane binding assay}

A membrane binding assay was developed to detect the adherence of salivary bacteria to selected oral microbes (bait organisms) immobilized on a solid support according to a previously described assay [9] with some modifications. Nitrocellulose membranes $(0.45 \mu \mathrm{m}$ pore size; GE Water and Process Tech) were cut into $4 \mathrm{~cm}^{2}$ pieces and each piece was placed in a sterile Petri dish (100 mm $\times 15 \mathrm{~mm}$, Fisher, USA). Bait organisms were harvested by centrifugation at $10000 \times g$ for $10 \mathrm{~min}$. Cell pellets were washed twice and resuspended in PBS buffer. The cell concentration was adjusted to $2 \times 10^{9}$ cells $\cdot \mathrm{mL}^{-1}$ and $50 \mu \mathrm{L}$ of the cell suspension was added to the centre of each nitrocellulose membrane. The nitrocellulose with immobilized bacteria (bait organism blot) was washed three times (15 min each) in PBS buffer containing $0.1 \%$ Tween 20 (PBST) to remove loosely bound organisms. The non-specific sites of the nitrocellulose were blocked by soaking in PBST with 5\% BSA, 5\% non-fat dry milk and $200 \mu \mathrm{g} \cdot \mathrm{mL}^{-1}$ fish sperm DNA. The membrane was further washed 3 times with PBS buffer (15 min each).

The test bacteria were harvested by centrifugation at $10000 \times g$ for $10 \mathrm{~min}$, washed twice and resuspended in PBS buffer to a final concentration of $10^{7}$ cells $\cdot \mathrm{mL}^{-1}$. Defined bacterial mixture was constructed by mixing at 
equal culture volumes different combinations containing three of the following four species: $F$. nucleatum, $S$. mitis, $P$. gingivalis or E. coli. $10 \mathrm{~mL}$ of the bacterial mixture was added into each Petri dish containing the nitrocellulose membrane with bait organism. After incubation for $2.5 \mathrm{~h}$ at $37{ }^{\circ} \mathrm{C}$ on a rotary shaker at $20 \mathrm{tilts} \cdot \mathrm{min}^{-1}$, unbound cells were decanted, and the blot was washed four times for $15 \mathrm{~min}$ each with PBST. The areas of the nitrocellulose containing the bait species were excised, the bound organisms were washed off by $0.2 \mathrm{~mol} \cdot \mathrm{L}^{-1}$ glycine $(\mathrm{pH}=2.7)$ and collected. Nitrocellulose membrane without base organism blot was used as blank control.

\section{PCR-DGGE analysis}

Total genomic DNA of bacteria was isolated using the QIAamp $^{\text {TM }}$ DNA Micro Kit (QIAGEN, USA). DNA quality and quantity were measured by a UV spectrophotometer at $260 \mathrm{~nm}$ and $280 \mathrm{~nm}$ (Spectronic Genesys ${ }^{\mathrm{TM}}$, Spectronic Instrument, Inc., Rochester, NY).

Bacterial 16S ribosomal RNA (rRNA) genes were amplified by PCR as described previously [21]. In brief, the universal primer set, Bac1 (5'-CGCCCGCCGCGC CCCGCGCCCGTCCCGCCGCCCCCGCCCGACTAC GTGCCAGCAGCC-3') [22] and Bac2 (5'-GGACTACC AGGGTATCTAATCC-3') was used to amplify an approximately 300 base pair (bp) internal fragment of the 16S rRNA gene. Each $50 \mu \mathrm{L}$ PCR contained $100 \mathrm{ng}$ purified genomic DNA, $40 \mathrm{pmol}$ of each primer, $200 \mu \mathrm{mol} \cdot \mathrm{L}^{-1}$ dNTPs, $4 \mathrm{mmol} \cdot \mathrm{L}^{-1} \mathrm{MgCl}_{2}, 5 \mu \mathrm{L} 10 \times$ PCR buffer, and 2.5 U Taq DNA polymerase (Invitrogen). Cycling conditions were $94{ }^{\circ} \mathrm{C}$ for $3 \mathrm{~min}$, followed by 30 cycles of $94{ }^{\circ} \mathrm{C}$ for $1 \mathrm{~min}, 56^{\circ} \mathrm{C}$ for $1 \mathrm{~min}$ and $72{ }^{\circ} \mathrm{C}$ for $2 \mathrm{~min}$, with a final extension period of $5 \mathrm{~min}$ at $72{ }^{\circ} \mathrm{C}$. The resulting PCR products were evaluated by electrophoresis in $1 \%$ agarose gels.

Polyacrylamide gels at an $8 \%$ concentration were prepared with a denaturing urea/formamide gradient between $40 \%$ [containing $2.8 \mathrm{~mol} \cdot \mathrm{L}^{-1}$ urea and $16 \%(\mathrm{~V} / \mathrm{V})$ formamide] and $60 \%$ [containing $4.2 \mathrm{~mol} \cdot \mathrm{L}^{-1}$ urea and $24 \%(V / V)$ formamide]. Approximately $300 \mathrm{ng}$ of the PCR product was applied per well. The gels were submerged in $1 \times \mathrm{TAE}$ buffer $\left(40 \mathrm{mmol} \cdot \mathrm{L}^{-1}\right.$ Tris base, $40 \mathrm{mmol} \cdot \mathrm{L}^{-1}$ glacial acetic acid, $1 \mathrm{mmol} \cdot \mathrm{L}^{-1}$ ethylenediaminetetraacetic acid) and the PCR products were separated by electrophoresis for $17 \mathrm{~h}$ at $58{ }^{\circ} \mathrm{C}$ using a fixed voltage of $60 \mathrm{~V}$ in the Bio-Rad DCode System (Bio-Rad Laboratories, Inc., Hercules, CA). After electrophoresis, the gels were rinsed and stained for $15 \mathrm{~min}$ in $1 \times \mathrm{TAE}$ buffer containing $0.5 \mu \mathrm{g} \cdot \mathrm{mL}^{-1}$ ethidium bromide, followed by $10 \mathrm{~min}$ of destaining in $1 \times \mathrm{TAE}$ buffer. DGGE profile images were digitally recorded using the Molecular Imager Gel Documentation system
(Bio-Rad Laboratories).

\section{Sequencing of PCR products}

PCR products were excised from the DGGE gel, eluted into $20 \mu \mathrm{L}$ sterile $\mathrm{dH}_{2} \mathrm{O}$ as preciously described [23] and re-amplified with the $\mathrm{Bac} 1 / \mathrm{Bac} 2$ universal primers. The resulting PCR products were purified and sequenced at the UCLA sequencing and genotyping core facility.

\section{Sequence analysis}

The obtained partial 16S rRNA gene sequences were used to BLAST search the HOMD (http://www.homd. org) and NCBI (http://www.ncbi.nlm.nih.gov) databases. Sequences with $98 \%$ to $100 \%$ identity to those deposited in the public domain databases were considered to be positive identification of taxa.

\section{Coaggregation assay}

Coaggregation assays were performed to determine the interbacterial binding in suspension as previously described [18]. In brief, bacteria were collected and resuspended in coaggregation buffer containing $150 \mathrm{mmol} \cdot \mathrm{L}^{-1}$ $\mathrm{NaCl}, 1 \mathrm{mmol} \cdot \mathrm{L}^{-1}$ Tris $/ \mathrm{HCl} \mathrm{pH} 8,0.1 \mathrm{mmol} \cdot \mathrm{L}^{-1} \mathrm{CaCl}_{2}$, $0.02 \% \mathrm{NaN}_{3}$, and $0.1 \mathrm{mmol} \cdot \mathrm{L}^{-1} \mathrm{MgCl}_{2}$ [14]. The concentration of the bacterial suspensions was adjusted to $2 \times 10^{9}$ cells $\cdot \mathrm{mL}^{-1}$ and equal volumes $(0.2 \mathrm{~mL})$ were mixed in sterile test tubes by vortexing for $30 \mathrm{~s}$ (Vortex -2 genie, Scientific industries, USA), followed by gentle agitation for $10 \mathrm{~s}$. After allowing the mixtures to settle for $30 \mathrm{~s}$, the degree of coaggregation was scored using a semiquantitative assay originally described by Cisar et al. [14]. Tubes containing: (a) coaggregation buffer solution alone; and (b) each test bacterial suspension alone, served as negative control.

\section{Results}

Membrane binding assay allowed identification of multiple co-adherence partners for single bacterial species from a defined microbial community

For comparison with the classical coaggregation assay, defined microbial communities containing known bacterial species were used to test the reliability and reproducibility of the membrane-based assay used in this study.

The coaggregation profiles of $F$. nucleatum, S. mitis, $P$. ginigivalis and $E$. coli with the strains used in this study were tested with the standard coaggregation assay (Table 1). Each species was then used as the "bait" organism in the membrane assay and incubated with the corresponding mixtures of the remaining species. PCRDGGE analysis revealed that the membrane assay 
reflected the results of the coaggregation assay (Figure $1)$. When $F$. nucleatum was used as bait organism, both S. mitis and P. gingivalis were bound, while $S$. mitis and $P$. gingivalis only interacted with $F$. nucleatum. E. coli was unable to interact with any of the oral species present in the defined community.

Table 1 Coaggregation properties of $F$. nucleatum, S. mitis, $P$. gingivalis and E. coli

\begin{tabular}{lcccc}
\hline \multirow{2}{*}{ species } & \multicolumn{4}{c}{ Coaggregation score $^{*}$ with } \\
\cline { 2 - 5 } & F. nucleatum & S. mitis & P. gingivalis & E. coli \\
\hline F. nucleatum & 0 & & & \\
S. mitis & 4 & 0 & & \\
P. gingivalis & 3 & 0 & 0 & \\
E. coli & 0 & 0 & 0 & 0 \\
\hline
\end{tabular}

*0: no visible coaggregation; 1 : small coaggregates, remaining in solution; 2: larger coaggregates, not immediately falling out of solution; 3: larger coaggregates, immediately clearing, slightly turbid suspension; 4: large coaggregates, setting immediately.

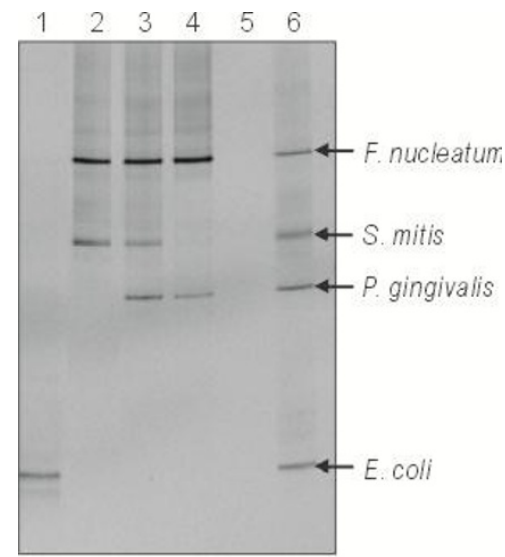

Figure 1 Co-adherence profiles of selected species with a defined bacterial mixture. PCR-DGGE analysis showiing lane 1: E. coli as bait organism incubated with a mixture containing $F$. nucleatum, $S$. mitis and $P$. gingivalis; lane 2: $S$. mitis as bait organism incubated with a mixture containing $F$. nucleatum, $E$. coli and $P$. gingivalis; lane 3: $F$. nucleatum as bait organism incubated with a mixture containing $S$. mitis, E. coli and $P$. gingivalis; lane 4: $P$. gingivalis as bait organism incubated with a mixture containing $F$. nucleatum, $E$. coli and S. mitis; lane 5: control nitrocellulose membrane without bait organism incubated with experimental organisms; lane 6: ladder containing $F$. nucleatum, $S$. mitis, $P$. gingivalis and $E$. coli. Two biological replicates were performed and a representative gel image is shown.
Previous study indicated that coaggregation of $F$. nucleatum with certain oral bacterial species could be inhibited by arginine [24]. Our coaggregation assay confirmed this observation, showing that the presence of $50 \mathrm{mmol} \cdot \mathrm{L}^{-1}$ arginine completely abolished the coaggregation between $F$. nucleatum and the test organisms (Table 2). This was also accurately reflected by the membrane binding assay, which showed that in the presence of arginine, bands corresponding to $S$. mitis and $P$. gingivalis were no longer observed in the DGGE profile of the interacting partner species of $F$. nucleatum (Figure 2).

Table 2 Coaggregation of $F$. nucleatum with $S$. mitis, $P$. gingivalis and $E$. coli in the presence/absence of arginine

\begin{tabular}{lcccc}
\hline \multicolumn{1}{c}{ species } & \multicolumn{4}{c}{ Coaggregation score $^{*}$ with } \\
\cline { 2 - 5 } & F. nucleatum & S. mitis & P. gingivalis & E. coli \\
\hline $\begin{array}{l}\text { F. nucleatum } \\
\text { Without arginine }\end{array}$ & 0 & 4 & 3 & 0 \\
$\begin{array}{l}\text { F. nucleatum } \\
\text { With arginine }^{* *}\end{array}$ & 0 & 0 & 0 & 0 \\
\hline
\end{tabular}

* see legend in table 1 for details. ** $50 \mathrm{mmol} \cdot \mathrm{L}^{-1}$ arginine.

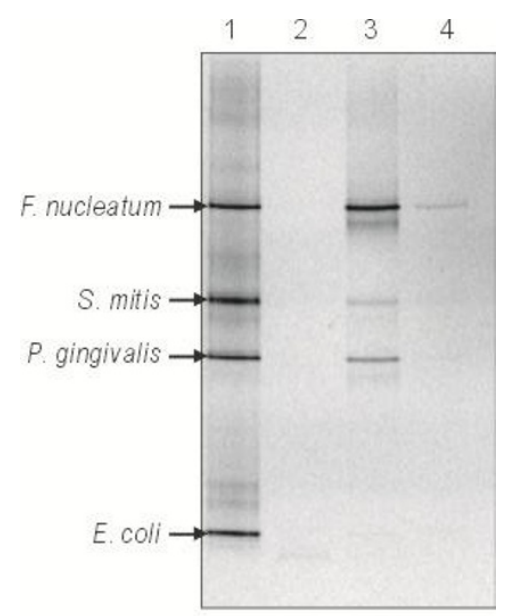

Figure 2 Co-adherence profiles of $F$. nucleatum with a defined bacterial mixture in the presence and absence of arginine. PCR-DGGE analysis showing lane 1: ladder containing F. nucleatum, S. mitis, P. gingivalis and E. coli; lane 2: control nitrocellulose membrane without bait organism incubated with experimental organisms; lane 3: F. nucleatum as bait organism incubated with a bacterial mixture containing $S$. mitis, $E$. coli and $P$. gingivalis in the absence of arginine; lane 4: F. nucleatum as bait organism incubated with a mixture containing $S$. mitis, $E$. coli and $P$. gingivalis in the presence of $50 \mathrm{mmol} \cdot \mathrm{L}^{-1}$ arginine. Two biological replicates were performed and a representative gel image is shown. 
Analysis of the co-adherence profiles of selected oral bacteria

To further test the effectiveness of the new approach in identifying the co-adherence partners from a complex microbial community, we chose $F$. nucleatum and $S$. mutans, two clinically relevant oral bacterial species, as the bait organisms to identify their co-adherence partners from salivary microbial flora cultivated in SHI medium. The membrane binding assay revealed that these two selected oral bacterial species displayed distinct coadherence profiles with salivary bacteria (Figure 3 ). The co-adherence partners of $S$. mutans were limited to Fusobacterium sp., Haemophilus sp. and Streptococcus sp., while $F$. nucleatum adhered to a variety of bacterial species including Fusobacterium sp., Gemella sp., Granulicatella sp., Haemophilus sp., Neisseria sp., Peptostreptococcus sp., Porphyromonas sp., Prevotella sp., and Streptococcus sp.. These data were consistent

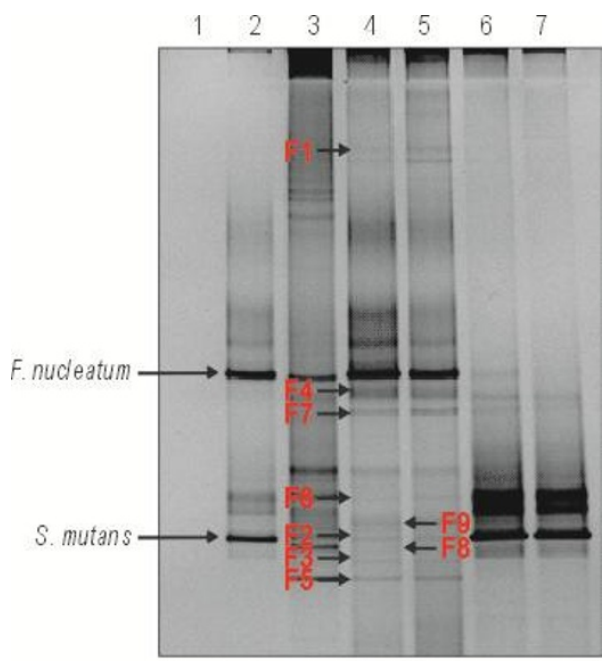

Figure 3 Co-adherence profiles of $F$. nucleatum and $S$. mutans with SHI medium cultured salivary microbial community. PCR-DGGE analysis showing lane 1: control nitrocellulose membrane without bait organism incubated with experimental organisms; lane 2: ladder of bait organisms F. nucleatum and S. mutans; lane 3: SHI medium cultured salivary bacteria; lane 4 and 5 : two independent replicates of $F$. nucleatum as bait organism incubated with $\mathrm{SHI}$ medium cultivated salivary bacteria; lane 6 and 7: two independent replicates of $S$. mutans as bait organism incubated with SHI medium cultivated salivary bacteria. Sequencing results are indicated: F1-Fusobacterium sp.; F2-Gemella sp.; F3Granulicatella sp.; F4-Neisseria sp.; F5-Peptostreptococcus sp.; F6-Porphyromonas sp.; F7, F8, F9-unclassified uncultured bacteria. with previously reported coaggregation assay result [25-26]. More importantly, our assay identified several previously unculturable species that adhered to $F$. nucleatum in the membrane binding assay.

Oral bacterial species displayed less co-adherence with mice gut bacteria

Previous studies employing the standard coaggregation assay have shown that bacteria from different microbial communities exhibit less interbacterial binding than species originating from the same community [27]. Therefore, we investigated the co-adherence profiles of $F$. nucleatum and $S$. mutans with a foreign flora using cultivated mice gut flora as a model community. Neither oral bacterial species exhibited significant co-adherence with mice gut bacteria. The only gut species found to bind to $F$. nucleatum was identified as Lactobacillus sp. (Figure 4).

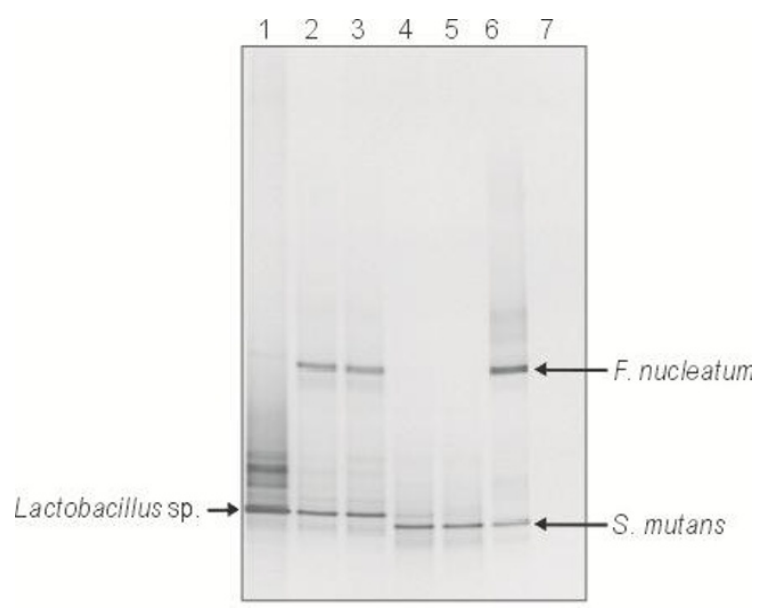

Figure 4 Co-adherence profiles of $F$. nucleatum and $S$. mutans with $\mathrm{BHI}$ cultivated mice fecal bacteria. PCRDGGE analysis showing lane 1: BHI cultured mice fecal bacteria; lane 2 and 3: two independent replicates of $F$. nucleatum as bait organism incubated with $\mathrm{BHI}$ cultivated mice fecal bacteria; lane 4 and 5: two independent replicates of $S$. mutans as bait organism incubated with $\mathrm{BHI}$ cultivated mice fecal bacteria; lane 6: ladder of bait organisms F. nucleatum and S. mutans; lane 7: control nitrocellulose membrane without bait organism incubated with experimental organisms.

\section{Discussion}

The bacterial residents within oral microbial community display extensive interactions with each other [10]. 
The physical binding between two oral bacterial species in suspension was first documented in 1970 [15]. It is thought to be a highly specific recognition and adhesion between surface components of different bacterial species. This interaction has also been suggested to play a pivotal role in establishing the highly structured multispecies oral plaque communities [28-29]. The coaggregation assay in which two test microbes were mixed in suspension and the interbacterial interaction was determined by monitoring the precipitation as a result of formation of coaggregates, has been the standard assay to investigate the physical interaction between different bacterial interspecies [30-31]. By immobilizing one bacterial species on a nitrocellulose membrane as "bait" organism and adding radio-labeled testing species, Lamont et al. developed a membrane-based binding assay to measure the binding between two bacterial species [9]. Due to its higher sensitivity, this new assay revealed novel interbacterial binding that could not be shown by the standard coaggregation assay. However, like other methods, this membrane assay is limited to bacterial species that can be grown in culture, and only examines bacterial co-adhesion at a dual species level. Since over 600 bacterial species reside in the human oral cavity [3], the current approaches of investigating bacterial interaction one-on-one makes the systematic study of interbacterial binding of oral bacteria a difficult task. Furthermore, more than $50 \%$ of the oral microflora are unculturable and cannot to be characterized using these types of assays [32].

To overcome these limitations, we developed a new approach by combining the membrane binding assay with PCR-DGGE profiling. Nitrocellulose membranes were used for immobilizing "bait" organisms, which has been shown to provide ease of manipulation for studying interspecies co-adhesion $[9,33]$, while the addition of PCR-DGGE enhanced the analyzing capacity. As a community-based molecular technique for analyzing microbial community profiles, PCR-DGGE is capable of detecting multiple bacterial species within the same microbial sample [10]. Our data derived from defined bacterial mixtures demonstrated that the newly developed approach was able to identify co-adherence partners which were verified by the classical coaggregation assay (Table 1 and Figure 1). The addition of arginine resulted in the same outcome for both assays (Table 2 and Figure 2 ), indicating that similar recognition and adhesion mechanisms are involved in the interbacterial binding under the different experimental setups.

F. nucleatum and S. mutans were selected as oral representative bacterial species to study their co-adherence profiles within a salivary microbial community in more detail. Due to its ability to coaggregate with a variety of oral bacterial species, $F$. nucleatum is considered a "bridging organism" and has been suggested to play an important role in the development of oral microbial communities $[4,8,34]$, while $S$. mutans is one of the early colonizers which can adhere to teeth surface by producing glucans and is generally regarded as a cariogenic bacterium [35-37]. We hypothesized that considering the different roles of these two bacterial species in dental plaque formation they could possess different co-adherence ability and have distinct interacting partners. Our data revealed that these two bacterial species indeed displayed different co-adherence profiles, with $F$. nucleatum showing a far more diverse panel of interacting partner (Figure 3) which is consistent with its role as a bridging organism in biofilm architecture. More importantly, the coupling of the membrane binding assay with PCR-DGGE profiling enhanced the identification capacity of co-adherence partners from a complex microbial community and allowed simultaneous detection of multiple bacterial interacting partners of $F$. nucleatum in the same experiment (Figure 3). Furthermore, by using the recently developed SHI medium, which is much less selective than other media in cultivating oral microbes [19], we were able to broaden the spectrum of microorganisms that adhere to $F$. nucleatum by discovering interactions with currently unculturable and uncharacterized bacteria.

Previous studies showed that bacteria from different origins were less likely to have direct physical binding as revealed by coaggregation assay [27]. Using BHI cultivated mice gut microbial community [20] we further tested the interbacterial binding between oral species and gut flora. Our data are in agreement with the previous reports, showing that the only mice gut bacterial species displaying binding capability with $F$. nucleatum was Lactobacillus sp. (Figure 4). Lactobacilli belong to the few gastrointestinal tract associated bacteria that are found in both oral cavity and intestinal tract of human and mice.

\section{Conclusion}

The new approach developed in this study combines the advantages of the membrane binding assay and PCR-DGGE profiling technique and allows for efficient analysis of co-adherence profiles of oral bacterial species. Our data demonstrated its effectiveness in identifying multiple co-adherence partners from complex microbial community and will greatly enhance our ability to systematically study the interbacterial binding within the community. Furthermore, this assay could be expanded 
to search for binding partners within original environmental samples, such as saliva, to identify unculturable bacterial species and expand our knowledge regarding bacterial interactions.

\section{Acknowledgement}

We would like to thank Dr. Li-hong Guo, Aida Kolesnikova, Dr. Ian McHardy, Dr. Sung-Hoon Lee, Dr. Hong-wei Pan, Dong-li Wang, Jing Wang, Ting-xi Wu and Zhe Yang for helpful discussion. This work was supported by Chinese State Scholarship Fund to R. Wang and US National Institutes of Health (NIH) Grants DE020102 and GM95373 to W. Shi.

\section{References}

1 Paster BJ, Boches SK, Galvin JL, et al. Bacteria diversity in human subgingival plaque. J Bacteriol 2001; 183: 37703783.

2 Aas JA, Paster BJ, Stokes LN, Olsen I, Dewhirst FE. Defining the normal bacterial flora of the oral cavity. J Clin Microbiol 2005; 43: 5721-5732.

3 Dewhirst FE, Chen $\mathrm{T}$, Izard $\mathrm{J}$, et al. The human oral microbiome. J Bacteriol 2010; 192: 5002-5017.

4 Listgarden MA. The structure of dental plaque. Periodontol 2000 1994; 5: 52-65.

5 Socransky SS. Criteria for the infectious agents in dental caries and periodontal disease. J Clin Periodontol 1979; 6: 16-21.

6 Dahlen G. Role of suspected periodontopathogens in microbiological monitoring of periodontitis. Adv Dent Res 1993; 7 : 163-174.

7 Marsh PD. Microbial ecology of dental plaque and its significance in health and disease. Adv Dent Res 1994; 8: 263-271.

8 Tatsuji N, Takeyoshi K. Microbial etiology of periodontitis. Periodontol 2000 2004; 36: 14-26.

9 Lamont RJ, Rosan B. Adherence of mutant streptococci to other oral bacteria. Infect Immun 1990; 58: 1738-1743.

10 Kuramitsu HK, He X, Lus R, Anderson MH, Shi W. Interspecies interactions within oral microbial communities. Microbiol Mol Biol Rev 2007; 71: 653-670.

11 Gibbons RJ, van Houte J. Bacterial adherence in oral microbial ecology. Annu Rev Microbiol 1975; 29: 19-44.

12 Scheie AA. Mechanisms of dental plaque formation. $A d v$ Dent Res 1994; 8: 246-253.

13 Kolenbrander PE, Anderson RN, Blehert DS, et al. Communication among oral bacteria. Microbiol Mol Biol Rev 2002; 66: 486-505.

14 Cisar JO, Kolenbrander PE, McIntire FC. Specificity of coaggregation reactions between human oral streptococci and strains of Actinomyces viscosus or Actinomyces naeslundii. Infect Immun 1979; 24: 742-752.

15 Gibbons RJ. Adherent interactions which may affect microbial ecology in the mouth. J Dent Res 1984; 63: 378-385.

16 Malik A, Sakamoto M, Hanazaki S, et al. Coaggregation among nonflocculating bacteria isolated from activated sludge. Appl Envir Microbiol 2003; 69: 6056-6063.

17 Jenkinson HF, Lala HC, Shepherd MG. Coaggregation of streptococcus sanguis and other streptococci with Candida albicans. Infect Immun 1990; 58: 1429-1436.

18 Rickard AH, Mcbain AJ, Ledder RG, Hadley PS, Gilbert P Coaggregation between freshwater bacteria within biofilm and planktonic communities. FEMS Microbiol Lett 2003, 220: $133-140$.

19 Tian Y, He X, Torrelba M, et al. Using DGGE profiling to develop a novel culture medium suitable for oral microbial communities. Mol Oral Microbiol 2010; 25: 357-367.

20 He X, Tian Y, Guo L, et al. In vitro communities derived from oral and gut microbial floras inhibit the growth of bacteria of foreign origins. Microb Ecol 2010; 60: 665-676.

21 Li Y, Ku CY, Xu J, Saxena D, Caufield PW. Survey of oral microbial diversity using PCR based denaturing gradient gel electrophoresis. J Dent Res 2005; 84: 559-564.

22 Sheffield VC, Cox DR, Lerman LS, Myers RM. Attachment of a 40-base-pair $\mathrm{G}+\mathrm{C}$-rich sequence (GC-clamp) to genomic DNA fragments by the polymerase chain reaction results in improved detection of single-base changes. Proc Natl Acad Sci U S A 1989; 86: 232-236.

23 Siqueira JF Jr, Rôças IN, Rosado AS. Investigation of bacterial communities associated with asymptomatic and symptomatic endodontic infections by denaturing gradient gel electrophoresis fingerprinting approach. Oral Microbiol Immunol 2004; 19: 363-370.

24 Kaplan CW, Lux R, Haake SK, Shi W. The Fusobacterium nucleatum outer membrane protein $\mathrm{RadD}$ is an arginineinhibitable adhesin required for inter-species adherence and the structured architecture of multispecies biofilm. Mol Microbiol 2009; 71: 35-47.

25 Kolenbrader PE, Andersen RN, Moore LV. Coaggregation of Fusobacterium nucleatum, Selenomonas flueggi, Selenomonas infelix, Selenomonas noxia, and Selenomonas sputigena with strains from 11 genera of oral bacteria. Infect Immun 1989; 57: 3194-3203.

26 Bradshaw DJ, Marsh PD, Watson GK, Allison C. Role of Fusobacterium nucleatum and coaggregation in anaerobe survival in planktonic and biofilm oral microbial communities during aeration. Infect Immun 1998; 66: 4729-4732.

27 Ledder RG, Timperley AS, Friswell MK, Macfarlane S, McBain AJ. Coaggregation between and among human intestinal and oral bacteria. FEMS Microbiol Ecol 2008; 66: 630-636.

28 Bos R, van der Mei HC, Meinders JM, Busscher HJ. A 
quantitative method to study co-adhesion of microorganisms in a parallel plate flow chamber: basic principles of the analysis. J Microbiol Meth 1994; 20: 289-305.

29 Kolenbrander PE, Ganeshkumar N, Cassels FJ, Hughes CV. Coaggregation: specific adherence among human oral plaque bacteria. FASEB J 1993; 7: 406-413.

30 Min KR, Rickard AH. Co-aggregation by the freshwater bacterium Sphingomonas natatoria alters dual-species biofilm formation. Appl Environ Microbiol 2009; 75: 3987-3997.

31 Jabra-Rizk MA, Falkler WA, Merz WG, et al. Coaggregation of Candida dubliniensis with Fusobacterium nucleatum. J Clin Microbiol 1999; 37: 1464-1468.

32 Wilson MJ, Weightman A, Wade W. Applications of molecular ecology in the characterization of uncultured microorganisms associated with human disease. Rev Med Microbiol 1997; 8: 91-101.
33 Lamont RJ, Hersey SG, Rosan B. Characterization of the adherence of Porphyromonas gingivalis to oral streptococci. Oral Microbiol Immunol 1992; 36: 193-197.

34 Kolenbrander PE, Andersen RN, Holdeman LV. Coaggregation of oral Bacterioides species with other bacteria: central role in coaggragation bridges and competitions. Infect Immun 1985; 48: 741-746

35 Hamada S, Slade HD. Biology, immunology, and cariogenicity of Streptococcus mutans. Microbiol Rev 1980; 44: 331-384.

36 Loesche WJ. Role of Streptococcus mutans in human dental decay. Microbiol Rev 1986; 50: 353-380.

37 Ooshima T, Matsumura M, Hoshino T, et al. Contributions of three glucosyltransferases to sucrose-dependent adherence of Streptococcus mutans. J Dent Res 2001; 80: 1672-1677. 